\title{
BMJ Open Ultrasound assessment of diaphragmatic dysfunction as a predictor of weaning outcome from mechanical ventilation: a systematic review and meta-analysis
}

\author{
Zhicheng Qian, ${ }^{1}$ Ming Yang, ${ }^{2}$ Lin Li, ${ }^{3}$ Yaolong Chen ${ }^{4}$
}

To cite: Qian Z, Yang M, Li L, et al. Ultrasound assessment of diaphragmatic dysfunction as a predictor of weaning outcome from mechanical ventilation: a systematic review and meta-analysis. BMJ Open 2018;8:e021189. doi:10.1136/ bmjopen-2017-021189

- Prepublication history and additional material for this paper are available online. To view these files, please visit the journal online (http://dx.doi org/10.1136/bmjopen-2017021189).

Received 19 December 2017 Revised 9 May 2018 Accepted 5 September 2018

Check for updates

(c) Author(s) (or their employer(s)) 2018. Re-use permitted under CC BY-NC. No commercial re-use. See rights and permissions. Published by BMJ.

${ }^{1}$ Department of Intensive Care Unit, Affiliated Hospital of North Sichuan Medical College, Nanchong, China

${ }^{2}$ Department of Pharmacy, Affiliated Hospital of North Sichuan Medical College, Nanchong, China

${ }^{3}$ Department of Ultrasonography, Suining People's Hospital,

Suining, China

${ }^{4}$ Department of Evidence-based Medicine, Center of Lanzhou University, Lanzhou, China

Correspondence to Professor Yaolong Chen; chenyaolong21@163.com

\section{ABSTRACT}

Objective The aim of this systematic review was to assess the diaphragmatic dysfunction (DD) as a predictor of weaning outcome.

Background Successful weaning depends on several factors: muscle strength, cardiac, respiratory and metabolic. Acquired weakness in mechanical ventilation is a growing important cause of weaning failure. With the development of ultrasonography, DD can be evaluated with ultrasound in weakness patients to predict weaning outcomes.

Methods The Cochrane Library, PubMed, Embase, Ovid Medline, WanFang Data and CNKI were systematically searched from the inception to September 2017. Ultrasound assessment of DD in adult mechanical ventilation patients was included. Two independent investigators assessed study quality in accordance with the Quality Assessment of Diagnostic Accuracy Studies-2 tool. The primary outcome was diaphragmatic thickness and excursion in the weaning success and failure group. The secondary outcome was the influence of DD on weaning outcome.

Results Eleven studies involving a total of 436 patients were included. There were eight studies comparing diaphragmatic excursion (DE), five comparing the diaphragmatic thickening fraction (DTF) and two comparing DD between groups with and without successful weaning. Overall, the DE or DTF had a pooled sensitivity of $0.85(95 \% \mathrm{Cl} 0.77$ to 0.91$)$ and a pooled specificity of $0.74(95 \% \mathrm{Cl} 0.66$ to 0.80$)$ for predicting weaning success. There was high heterogeneity among the included studies $\left(l^{2}=80 \% ; p=0.0006\right)$. The rate of weaning failure was significantly increased in patients with DD (OR 8.82; 95\% Cl 3.51 to 22.13; $p<0.00001$ ).

Conclusions Both DE and DTF showed good diagnostic performance to predict weaning outcomes in spite of limitations included high heterogeneity among the studies. DD was found to be a predictor of weaning failure in critically ill patients.

\section{INTRODUCTION}

Weaning from mechanical ventilation (MV) is of paramount importance for patients with respiratory failure requiring MV. It is crucial because both premature discontinuation and delayed weaning are associated with

\section{Strengths and limitations of this study}

To our knowledge, this is the first review that systematically analyses the accuracy of diaphragm ultrasound for predicting weaning outcomes combined with role of diaphragmatic dysfunction for predicting weaning failure.

- Value of diaphragmatic excursion and diaphragmatic thickening fraction in different weaning outcome groups help to clinical application.

- High heterogeneous and small study effect should be mentioned in the results interpretation.

increased morbidity and mortality as well as high medical costs. ${ }^{1-4}$ However, it remains a challenge to identify reliable predictors of weaning outcome and to determine the timing of the initiation of the weaning process.

Difficulty with weaning from MV is attributable to many factors, such as acquired weakness, malnutrition, nervous system disease, cardiac insufficiency, infection and other diseases. ${ }^{5-7}$ There is increasing awareness that diaphragm weakness is common in patients undergoing MV and is likely a contributing factor of weaning failure. ${ }^{8-10}$ In the past few years, diaphragmatic dysfunction (DD) has been recognised as a common aetiology of weaning failure. ${ }^{811-13}$

With the development of critical care ultrasonography, doctors can use ultrasound to dynamically assess the causes of respiratory failure and weaning failure, which including pulmonary or extrapulmonary factors. Nowadays bedside assessment of diaphragmatic movements, such as amplitude, force and velocity of contraction, special patterns of motion and changes in diaphragmatic thickness during inspiration, has become readily available. ${ }^{11}$ It has been shown that the quantity and quality of the diaphragm and skeletal muscle as assessed by ultrasound are related 
to muscle strength and function; therefore, ultrasound is an effective method for the early detection and evaluation of acquired weakness in the intensive care unit (ICU). ${ }^{13}$

There are two proposed diaphragm sonographic predictors: the diaphragmatic excursion (DE) and diaphragm thickening fraction (DTF). The patients with supine position, DE is measured by ultrasonic probe in the right midline of the axillary and left axillary posterior line, respectively. In the M-mode, the distance between the highest and lowest point of the diaphragm movement is DE. DTF reflects variation in the thickness of the diaphragm during respiratory effort and is calculated as (thickness at end-inspiration-thickness at the end-expiration)/thickness at the end of the expiration. In the area of 8-10 ribs, the probe is placed between the axillary frontline and the midline perpendicular to the chest wall, the diaphragm is displayed. The hypoechoic diaphragm is located between the hyperechoic pleura and peritoneum.

With the growing evidence showing that DD plays an important role in the weaning process, we decided to systematically review the literature to assess the accuracy of diaphragm ultrasound for predicting weaning outcomes in critically ill adults and the role of DD to weaning failure. To our knowledge, nobody had reviewed that systematically analyses DD assessed by ultrasound for predicting weaning failure before.

\section{METHODS}

\section{Search strategy}

The electronic search of databases, including the Cochrane Library, PubMed, Embase, Ovid Medline, WanFang Data and CNKI, was performed by two independent investigators from their inception to September 2017, without language restrictions. The references of all retrieved articles were reviewed for potentially relevant manuscripts. The search strategy involved the use of the following keywords: ('diaphragm' or 'diaphragm dysfunction') and ('ultrasonography' or 'ultrasound' or 'echography') and ('weaning MV' or 'extubation') and ('factor' or 'predict'), as presented in online supplementary file 1 . The research string was developed to have the widest possible sensitivity, while the specificity was guaranteed by manual reviews of retrieved results as follows: one reviewer (YC) examined the titles and abstracts resulting from the electronic search to exclude articles that were obviously irrelevant. Two independent reviewers (ZQ and MY) examined the full text of the remaining studies. A third reviewer (YC) was employed to make the final decision when consensus could not be achieved.

\section{Inclusion and exclusion criteria}

Two authors independently identified and screened the search results for potentially eligible studies. Inclusion or exclusion of articles was determined by two independent investigators (ZQ and LL). Discrepancies were discussed and resolved by a third opinion (YC). The inclusion criteria were as follows: (1) Type of study: prospective or retrospective study involving human participants published in a peer-reviewed journal; (2) Population: subjected to invasive MV for at least 24 hours; (3) Intervention: diaphragm thickness and excursion measured by ultrasound during weaning process or around spontaneous breathing trial (SBT) and (4) Predefined outcomes: The primary outcome was the accuracy of diaphragm ultrasound for predicting weaning outcomes in critically ill adults. Weaning failure was defined broadly as SBT failure or the need for reintubation, or non-invasive MV or death within 48 hours. Weaning success was defined as the absence of criteria for failure. The secondary outcome was the influence of DD on the weaning outcome. Diagnostic criteria of DD by ultrasound were not unified so far. In Ali's study, ${ }^{12}$ DD was diagnosed when diaphragmatic thickness $<0.2 \mathrm{~cm}(2 \mathrm{~mm})$, a DTF inferior to $20 \%$ and or DE was $<15 \mathrm{~mm}(<1.5 \mathrm{~cm})$. In Kim's study, ${ }^{11} \mathrm{DD}$ was diagnosed by ultrasound if an $\mathrm{DE}<10 \mathrm{~mm}$ or a paradoxical movement was observed.

The exclusion criteria were as follows: (1) Abstracts, letters, editorials, expert opinions, reviews and case reports; (2) articles without sufficient data for the calculation of ORs or relative risk with 95\% CIs; (3) studies performed in settings other than critical care (ie, patients ventilated for elective surgery) and (4) maximal not mean $\mathrm{DE}$ as the ultrasound measurement.

\section{Patient and public involvement}

DD assessed by ultrasound helped to predict weaning outcome. But in clinical practice, we decided to weaning outcome or extubation based on SBT mostly. Patients were informed priorities, experience and preferences of the measurement. Patients were involved in the recruitment to and conduct of the study.

\section{Data extraction}

Two reviewers (ZQ and MY) extracted the data independently using a predefined data extraction form. Disagreements were resolved by a third opinion (YC). The data extracted included the study ID (the first author's name and publication year), country, study design, setting, DE and DTF in the weaning success and failure groups, true positives, true negatives, as well as false positives and false negatives of ultrasound parameters in predicting weaning failure. We also checked the online supplementary files and contacted the authors for more detailed information, if necessary.

\section{Quality assessment and publication bias}

The Quality Assessment of Diagnostic Accuracy Studies-2 (QUADAS-2) tool was employed to assess the risk of bias of diagnostic accuracy studies. The tool consisted of four domains of risk of bias, including patient selection, index test, reference standard and flow and timing. ${ }^{14}$ Publication bias was assessed by using a funnel plot, and plot asymmetry was considered to be suggestive of publication bias. 


\section{Statistical analysis}

The statistical analysis was performed using the Meta View statistical program within the Review Manager software (Rev Man V.5.3.4) using the Mantel-Haenszel random-and-fixed-effects model as well as Stata software (V.12.0) to compute the pooled sensitivity and specificity. Statistical heterogeneity across trials was assessed using Cochrane's $\chi^{2}$ test and the inconsistency test proposed by Higgins and Thompson. ${ }^{10}{ }^{15}$ Heterogeneity was significant when $\mathrm{p}<0.05$ and $/$ or $\mathrm{I}^{2}>50 \%$, and the random-effect model was used; if not, the fixed-effect model was applied. The data were also used to plot summary receiver operating characteristic (SROC) to establish the true positivity and false positivity (1-specificity) of each study. The closer the curve is to the upper left-hand corner, with the exact area under the curve (AUC) of the SROC curve plot, the better the overall accuracy of the test. In addition, subgroup analyses were performed to identify and explain the potential heterogeneity of the studies.
RESULTS

\section{Literature search results}

We initially identified 1019 citations from the databases and 8 citations from conferences. A total of 361 studies were obtained after removing duplicates. Of these, 343 articles were discarded after reviewing the abstracts. The full texts of the remaining 18 articles were examined in detail (figure 1). Seven articles did not provide valid data and were excluded (online supplementary file 2).

\section{Characteristics of the studies}

The main characteristics of the individual studies are summarised in table 1 . Of the 11 studies, 1 was retrospective and 10 were prospective in design. Ultrasound operator is nature blind to weaning outcome because of timeliness. They were all published between 2004 and 2017. The sample sizes ranged between 27 and 63. Five studies included patients from a medical ICU. Three studies involved mixed ICU patients. Two studies included respiratory ICU patients. One study included patients received tracheostomy in a high-dependency unit. Most

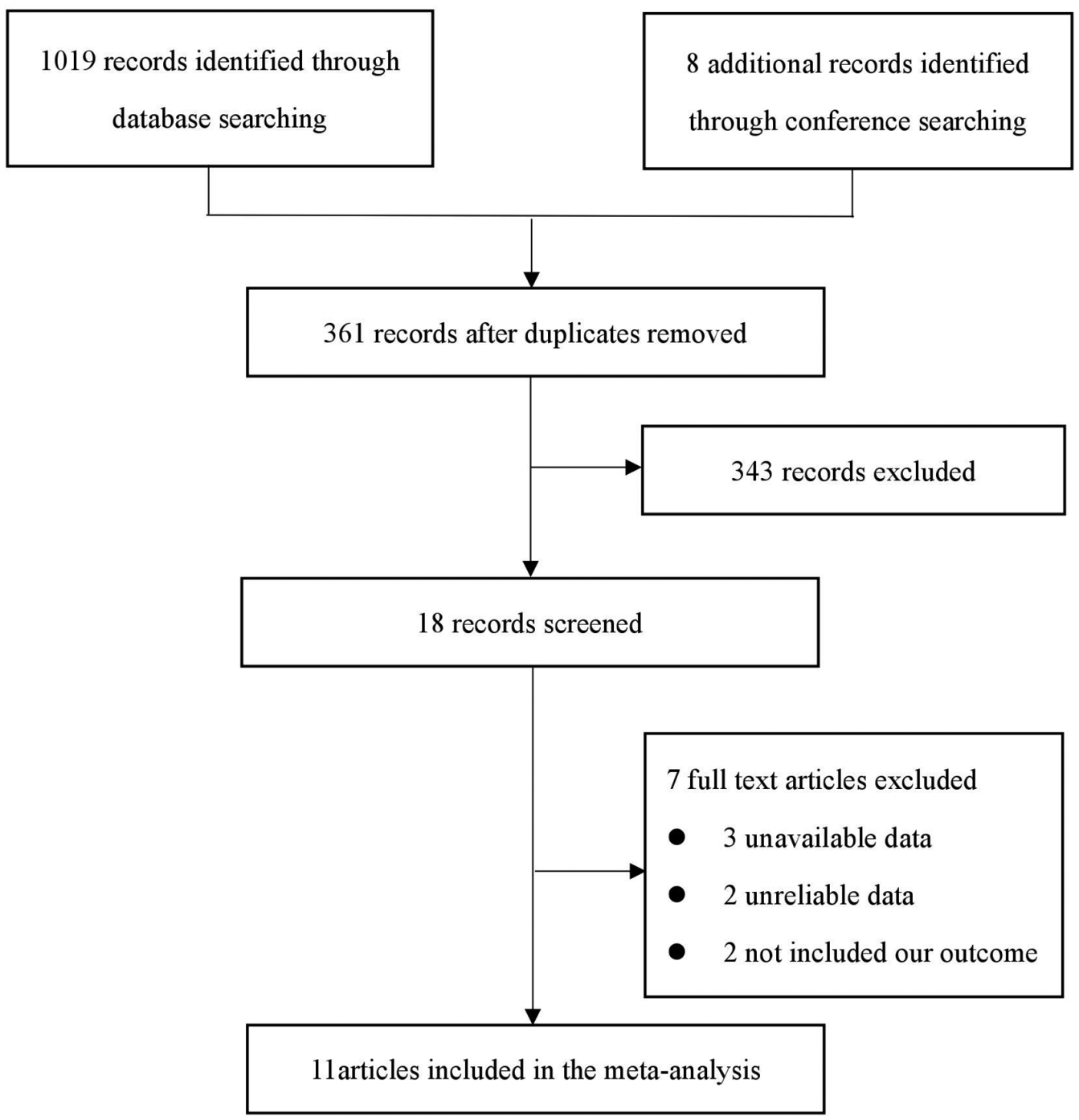

Figure 1 Selection of studies included in this meta-analysis. 


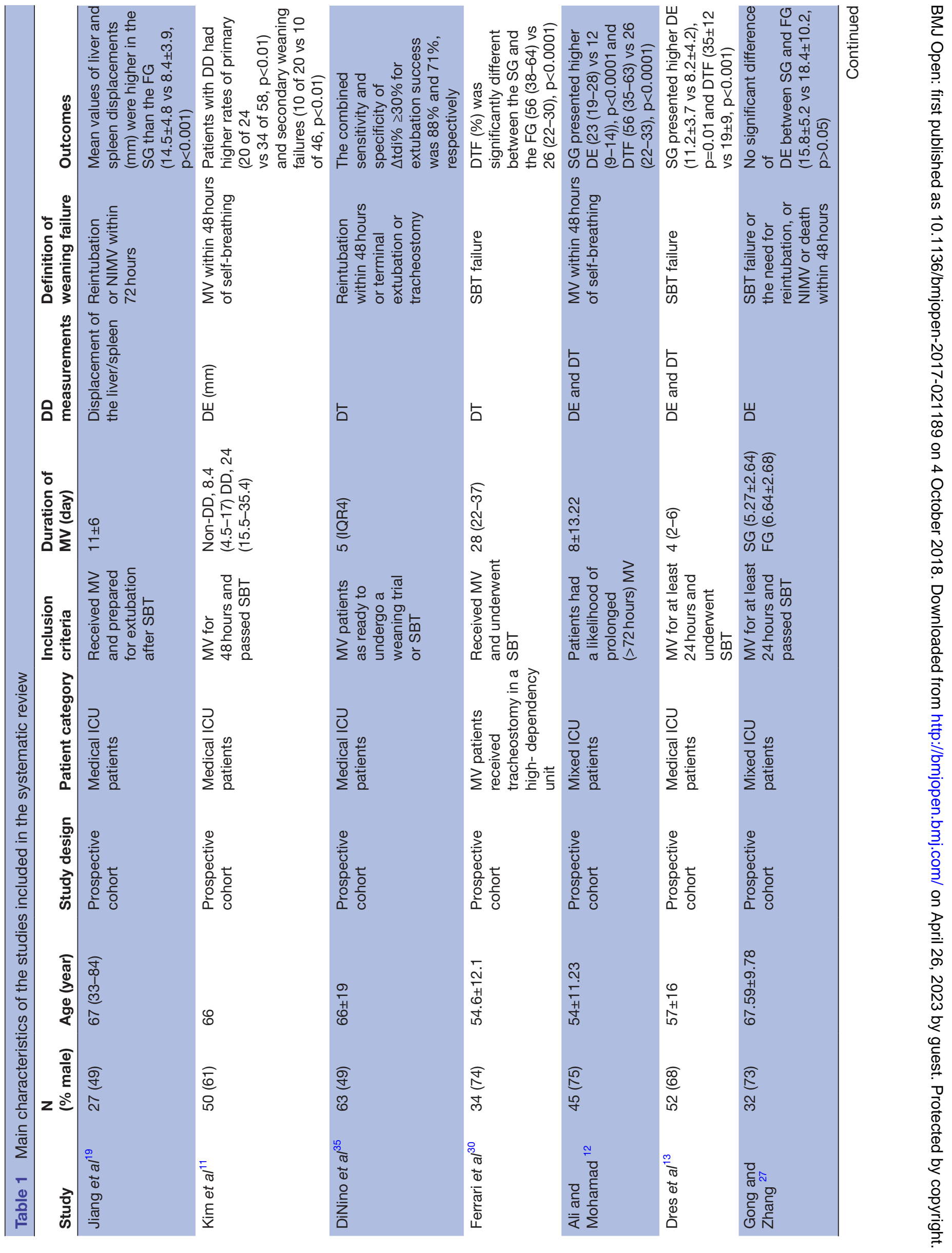




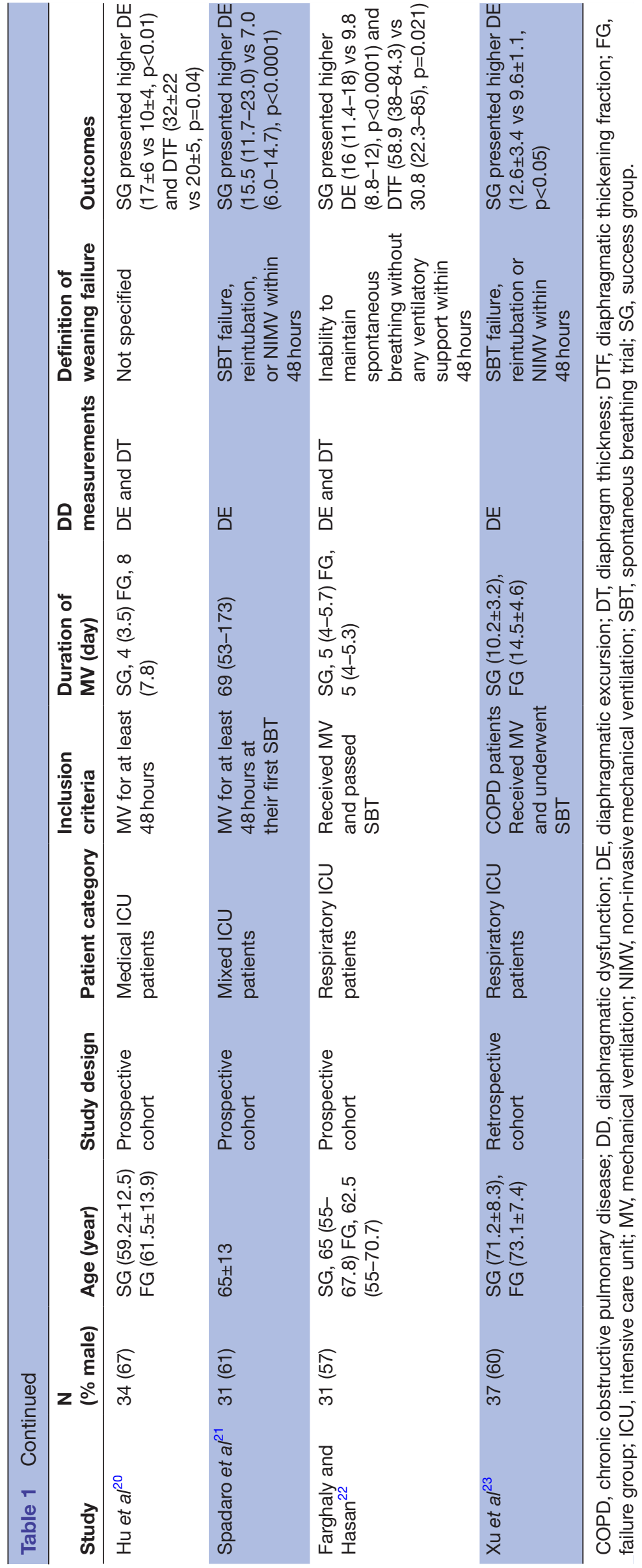




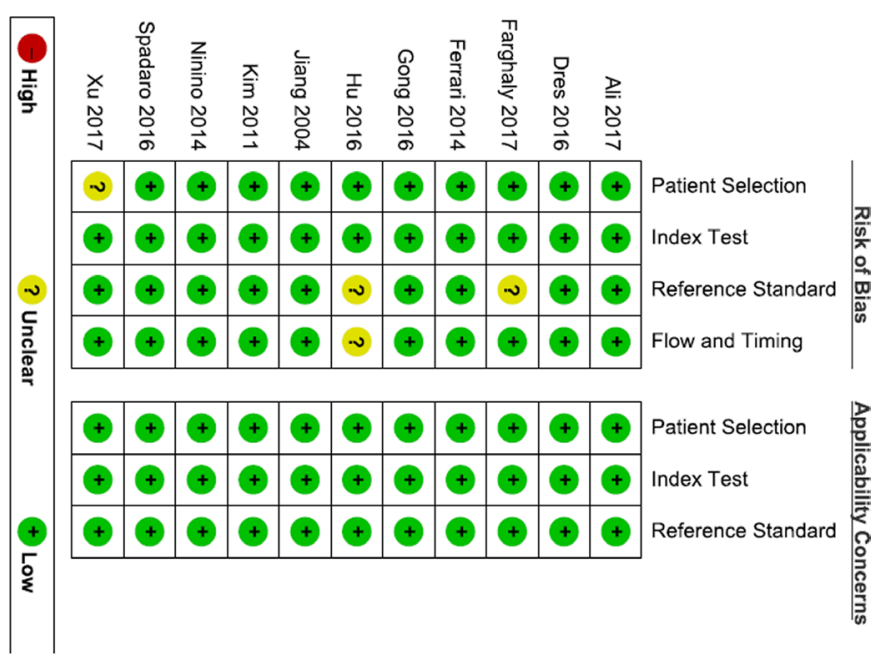

Figure 2 Assessment of risk of bias of studies: QUADAS-2 tool. QUADAS-2, Quality Assessment of Diagnostic Accuracy Studies-2.

of the observational studies showed a low risk of bias as assessed by the QUADAS-2 tool (figure 2). Visual analysis of the funnel plot was not suggestive of publication bias (figure 3).

\section{Diagnostic performance of DE and DTF to predict weaning success}

To predict weaning outcome from MV in medical or mixed ICU patients, either DE or DTF measurements performed during weaning process or around SBT were employed as the test index. Both indices showed good diagnostic performance to predict weaning outcomes (table 2). In Hu's study, cut-off value to predicting successful weaning was mean DE $>11 \mathrm{~mm}$, with the sensitivity and specificity were $92 \%$ and $100 \%$, particularly.

\section{Meta-analysis of DE or DTF to predict weaning success}

The DE or DTF to predict weaning success in each individual study is shown in figure 4. Overall, in 284 patients totally, the DE or DTF had a pooled sensitivity of 0.85

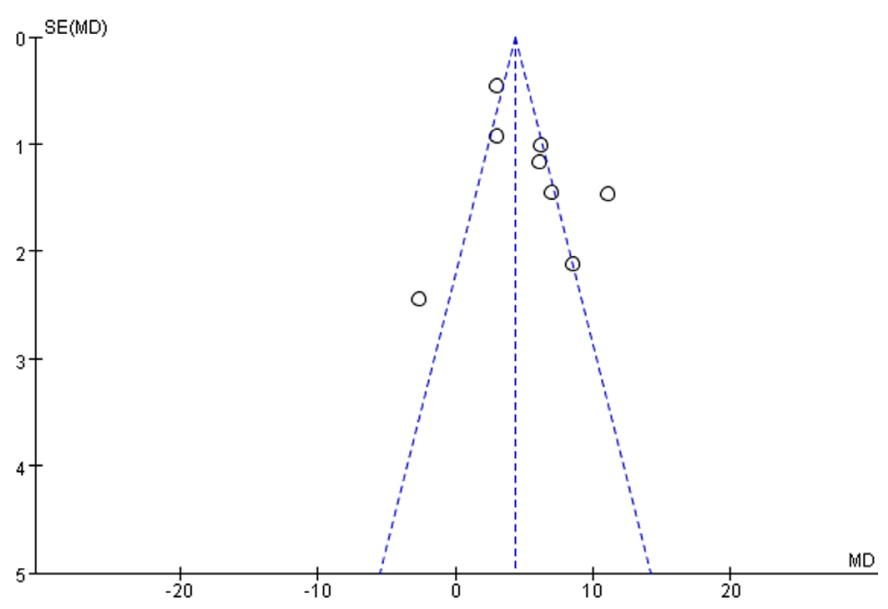

Figure 3 Funnel plot comparison of diaphragmatic excursion between different weaning outcomes by MD. MD, mean difference.
(95\% CI 0.77 to 0.91 ) and a pooled specificity of 0.74 (95\% CI 0.66 to 0.80$)$ for predicting weaning success. The SROC curve is shown in figure 5 .

\section{DE in different weaning outcome groups}

Eight articles involving 289 patients were included in this meta-analysis. DE was significantly associated with weaning success, with an increased excursion when compared with patients who had weaning failure (mean difference, 4.28; 95\% CI 3.62 to 4.94; $\mathrm{p}<0.00001)$. However, there was high heterogeneity among the component studies $\left(\mathrm{I}^{2}=87 \%\right.$; $\mathrm{p}<0.00001$, random-effects model) (figure 6).

\section{DTF in different weaning outcome groups}

The DTF was measured in five studies (all of them cohort studies). The pooled results showed that DTF in the weaning success group was significantly greater than that in the failure group (mean difference, 20.13; 95\% CI16.90 to 23.36; $\mathrm{p}<0.00001)$. However, there was remarkable heterogeneity among the studies $\left(\mathrm{I}^{2}=80 \%\right.$; $\mathrm{p}=0.0006$ ) (figure 7).

\section{DD in weaning failure}

Two studies compared the rate of weaning failure in the DD and the normal groups. The rate of weaning failure was significantly higher in the DD group (OR 8.82; 95\% CI 3.51 to $22.13 ; \mathrm{p}<0.00001)$. There was high heterogeneity among the studies $\left(I^{2}=84 \% ; p<0.00001\right.$; figure 8$)$.

\section{DISCUSSION}

In the past few years, diaphragm activity could not be accurately assessed at the bedside. Methods to assess diaphragmatic function often have a low sensitivity and specificity as in the case of chest X-rays, or they are invasive and difficult to obtain at the bedside as in the case of the gold-standard twitch magnetic phrenic nerve stimulation or measurement of transdiaphragmatic pressure with oesophageal and gastric balloons. ${ }^{16}$ Ultrasound has played an important role in the evaluation of diaphragmatic function, since it is non-invasive and readily available as well as allows repeated measurements.

Moreover, DE and DTF are two proposed diaphragm sonographic predictors. Our data show that the DE or DTF had a pooled sensitivity of 0.85 (95\% CI 0.77 to 0.91 ) and a pooled specificity of 0.74 (95\% CI 0.66 to $0.80)$ for predicting weaning success. In a recent systematic review, Llamas-Álvarez et $a l,{ }^{17}$ based on 19 studies, showed that DE, pooled sensitivity was $75 \%$ (95\% CI $65 \%$ to $85 \%$ ); pooled specificity, $75 \%$ (95\% CI $60 \%$ to $85 \%$ ) and DOR, 10 (95\% CI 4 to 24). Based on bivariate metaregression analysis, a significantly higher specificity for DTF and higher sensitivity for DE was detected in studies with applicability concerns. Earlier this year, Zambon et al ${ }^{18}$ reviewed usefulness of diaphragmatic ultrasound in ICU patients, which showed a good performance as weaning indexes. Compared with these previous studies, our new meta-analysis based on more widely studies especially 
from China, where ultrasound had developed fast in ICU, showed DE or DTF had a more sensitivity and equivalent specificity for predicting weaning outcomes. Beyond this, our review purposely compared the rate of weaning failure in the DD and the normal groups. To our knowledge, this is the first review that systematically analyses DD assessed by ultrasound for predicting weaning failure.

In our review, we found that DE and DTF were significantly associated with the weaning outcome, with increased DE and DTF in the weaning success group. Both DE and DTF measurements performed during a SBT in mechanically ventilated patients showed good performance as weaning indices. In this study, seven out of the eight studies reported significantly higher DE in the weaning success group as compared with the failure group. ${ }^{121319-23}$ The respiratory muscle capacity and load imbalance also contribute to extubation failure. ${ }^{24} 25$ The diaphragm plays a pivotal role in establishment of the respiratory muscle endurance and is considered as the main respiratory muscle as it generates approximately $70 \%$ of the total tidal volume during inspiration in healthy persons. ${ }^{26}$ Diaphragmatic movement is a final result of diaphragmatic strength as well as intrathoracic and intra-abdominal pressure. Evaluation of the DE by ultrasonography, therefore, may be an important tool to evaluate the respiratory endurance of a patient. However, one study ${ }^{27}$ has reported that DE was not statistically different between the success and failure groups $(15.8 \pm 5.2 \mathrm{~mm}$ vs $18.4 \pm 10.2 \mathrm{~mm}, \mathrm{p}>0.05$ ), but $\Delta \mathrm{DE}$ ( $30 \mathrm{~min}$ to $10 \mathrm{~min}$ during SBT) was higher in the failure group than in the success group $(1.07 \pm 0.64 \mathrm{~mm}$ vs $3.33 \pm 3.17 \mathrm{~mm}, \mathrm{p}<0.05)$. The difference may be attributable to the timing of the

Table 2 Diagnostic performance of DE and DTF

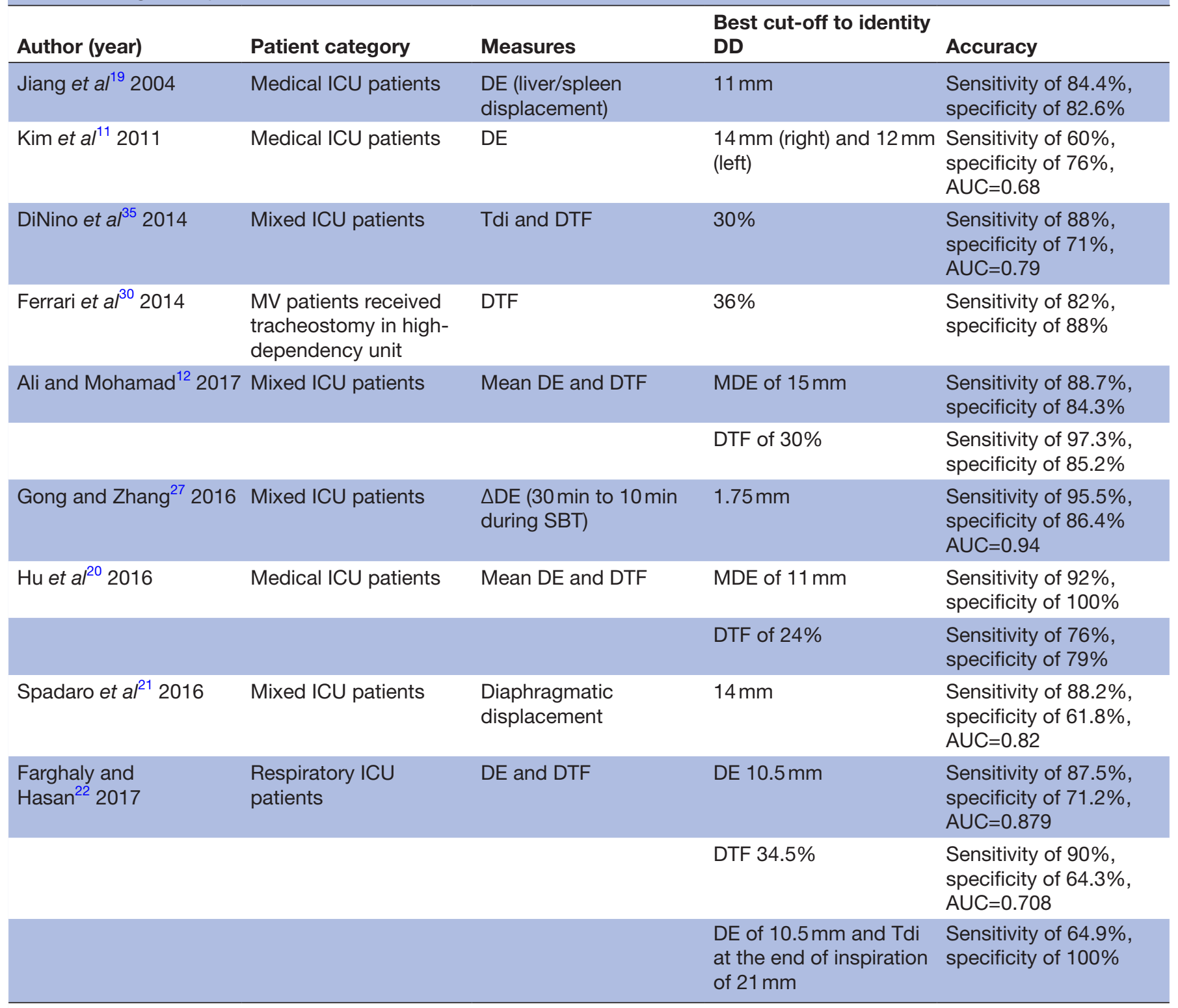

AUC, area under the curve; DD, diaphragmatic dysfunction; DE, diaphragmatic excursion; DTF, diaphragmatic thickening fraction; ICU, intensive care unit; MDE, mean diaphragmatic excursion; MV, mechanical ventilation; Tdi, thickness of diaphragm. 


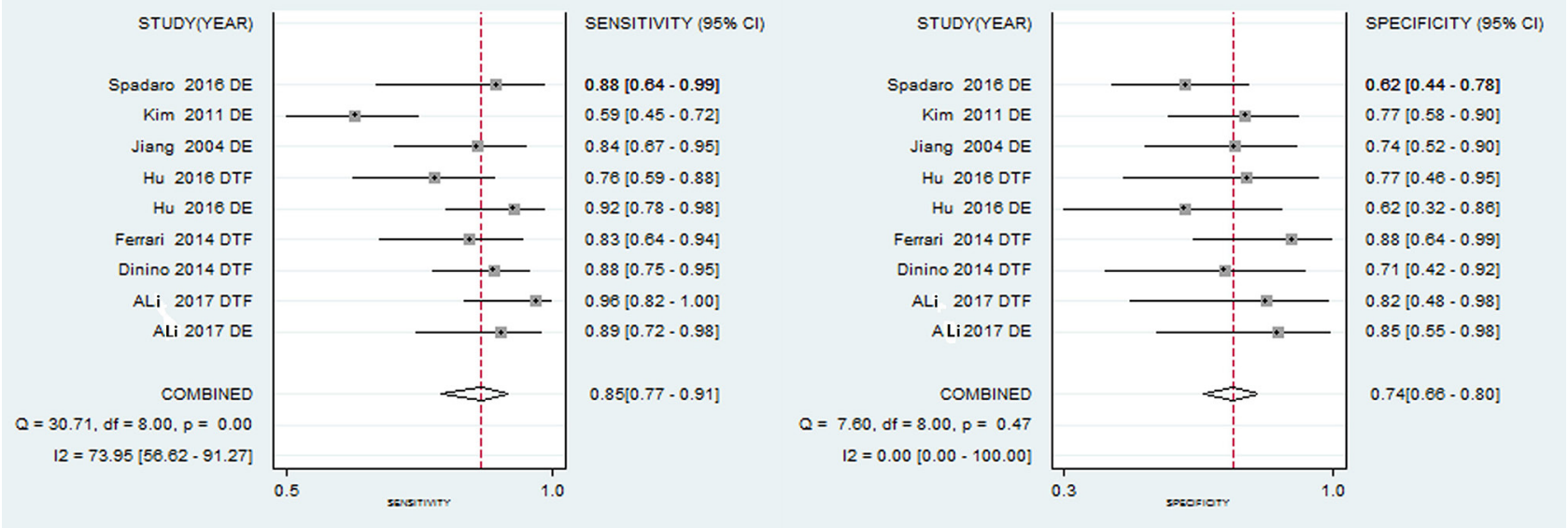

Figure 4 Diaphragmatic excursion (DE) or diaphragmatic thickening fraction (DTF) to predict weaning success.

measurements. While Gong and Zhang ${ }^{27}$ measured DE at 0,10 , and $30 \mathrm{~min}$ after the initiation of SBT, others measured it during $\mathrm{MV}^{12}$ or after $\mathrm{SBT}^{22}$ One study ${ }^{28}$ exclude in our system review because of using maximal not mean $\mathrm{DE}$ as the ultrasound measurement, mean values of maximal $\mathrm{DE}$ were significantly higher in patients who succeeded at their first weaning attempt $(4.1 \pm 2.1 \mathrm{vs}$ $3 \pm 1.8 \mathrm{~cm}, \mathrm{p}=0.04)$. Using a threshold of MDE $\leq 2.7 \mathrm{~cm}$, the sensitivity and specificity of diaphragmatic ultrasound in predicting weaning failure were $59 \%(39 \%-77 \%)$ and $71 \%(57 \%-82 \%)$ with an AUC at 0.65 (0.51-0.78). There was no significant difference between MDE values and Medical Research Council scores for predicting weaning failure $(p=0.73)$.

The diaphragm thickness evaluated by M-mode ultrasound is non-invasive and reproducible, which is useful

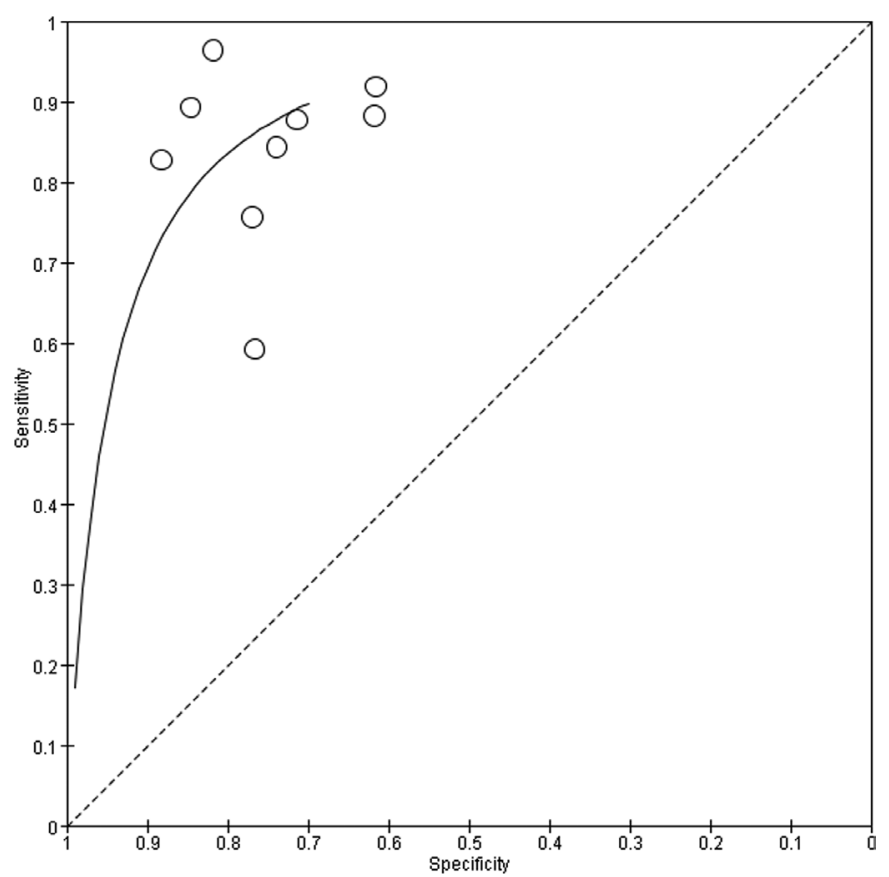

Figure 5 Summary of the receiver operating characteristic curve plotting sensitivity against specificity. to evaluate muscle function and its contribution to the respiratory workload. ${ }^{29}$ Because of the individual variability in the thickness of the diaphragm, DTF is considered to be a more reliable parameter for the evaluation of diaphragmatic function. In our systematic review, five studies reported a significantly higher DTF in the weaning success group, compared with the failure group. ${ }^{1213202230}$ However, there was significant heterogeneity among the component studies.

DD is common in mechanically ventilated patients at an early stage during their ICU stay ${ }^{8}$ and is responsible for delayed weaning as well as increased days of MV and mortality. ${ }^{31}{ }^{32}$ Although diagnostic criteria of DD by ultrasound were not unified so far, in pressure support ventilation, ${ }^{33}$ DTF and DE were respectively very strongly and moderately correlated to endotracheal pressure after phrenic nerve stimulation, which was regarded as gold standard to DD $(r=0.87, \mathrm{p}<0.001$ and $0.45, \mathrm{p}=0.001)$. In our meta-analysis, two studies compared the rate of weaning failure in the DD group and the normal group, despite some differences in the definition of DD. In one study, ${ }^{11}$ DD was defined as an excursion of less than $10 \mathrm{~mm}$ or a paradoxical movement. The other ${ }^{12}$ defined DD as a DTF of less than $20 \%$ and/or a DE of less than $15 \mathrm{~mm}$. However, no matter how DD was defined, it was consistent that DD was associated with an increased risk of weaning failure.

There were some limitations in the current study that must be acknowledged. First, the high level of heterogeneity in the study, which were when the ultrasound test was performed and the ununified definition of weaning failure. The component studies in this meta-analysis had been performed by researchers independently, thus, there were differences in the study population and interventions. The heterogeneity in the component studies was addressed with random-effects models. Second, The diagnostic criteria of DD by ultrasound were not unified so far. So in clinical practice, we should pay more attention to DE and DTF, not only emphasise in diagnostic criteria itself. Third, the number of studies included in this meta-analysis was small, especially for DTF and DD. An increased number 


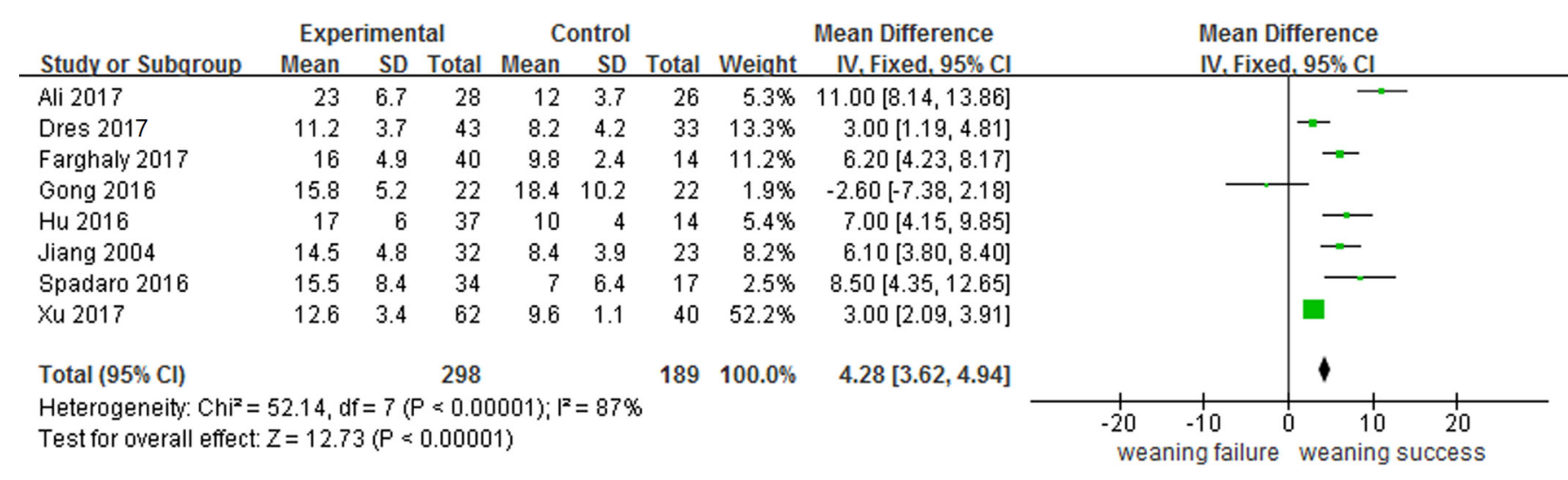

Figure 6 Mean difference of diaphragmatic excursion between the weaning failure and weaning success groups. IV, inverse variance.

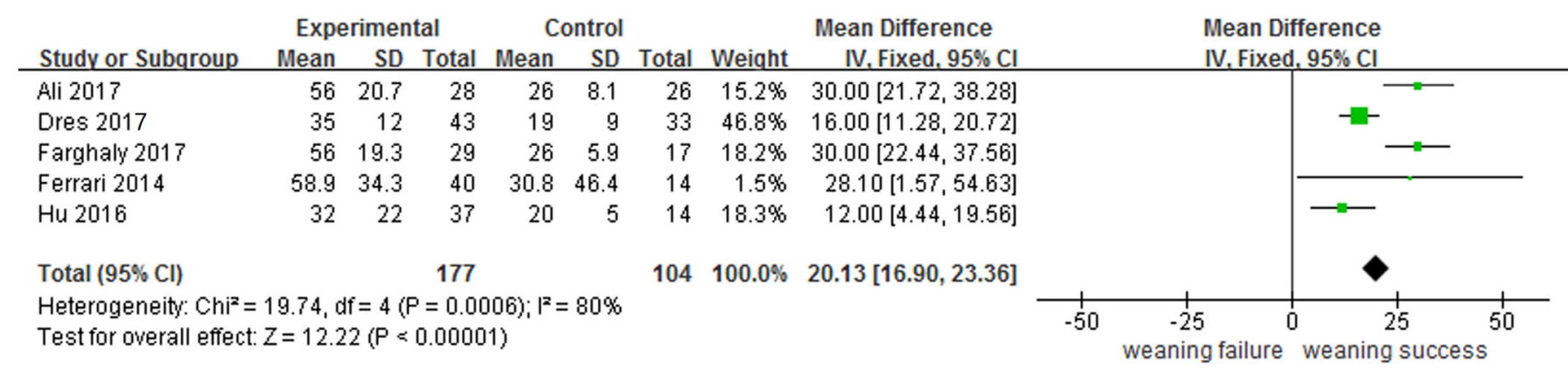

Figure 7 The diaphragmatic thickening fraction mean difference between the weaning failure and weaning success groups. IV, inverse variance.

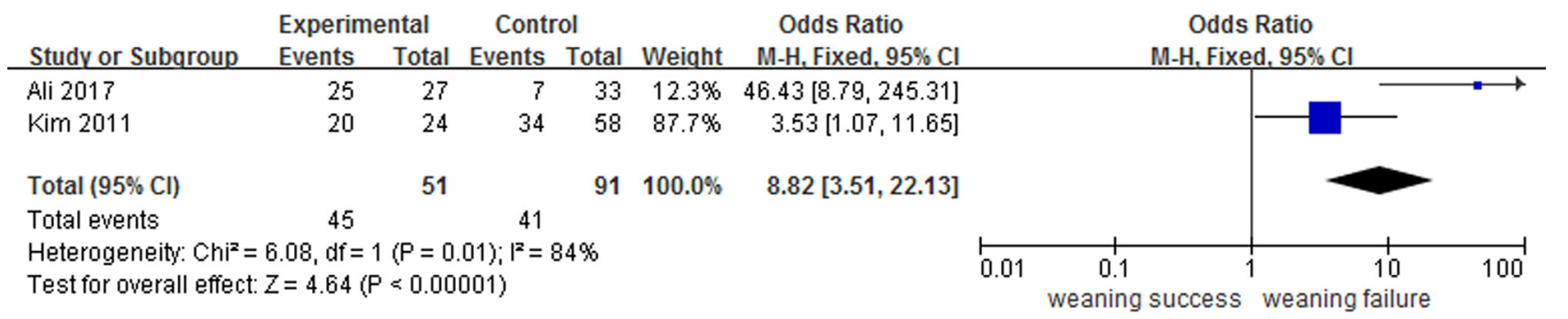

Figure 8 Weaning failure between the diaphragmatic dysfunction and normal groups. M-H, Mantel-Haenszel.

of high-quality studies need to be carried out in the future. Fourth, this study generally included small trials, which was subject to the 'small study effect'. ${ }^{34}$ Small trials are more likely to report larger beneficial effects than large trials in critical care medicine. Caution should be practised in the interpretation of meta-analyses involving small trials. Finally, this study demonstrated evidence of publication bias, which may be attributable to the fact that studies with negative results are less likely to be published.

\section{CONCLUSIONS}

In conclusion, diaphragmatic ultrasound may identify patients at risk of weaning failure. DD has been found to be a predictor of weaning failure in ICU patients. However, more studies are needed to standard the diagnostic criteria of DD with ultrasound and moreover, the diagnostic performance of DD to predict weaning outcome.
Contributors YC and ZQ contributed to review concept and design. ZQ and MY screened all search results and extracted the data independently using a predefined data extraction form. Inclusion or exclusion of articles was determined by ZQ and LL. ZQ and MY conducted the analysis and synthesis. YC was employed to make the final decision when consensus could not be achieved. LL contributed to ultrasonography interpretation. ZQ prepared the manuscript, and all authors revised it critically for important intellectual content and approved the final manuscript.

Funding The authors declared no specific grant for this research from any funding agency in the public, commercial or not-for-profit sectors.

Competing interests The authors declared no competing interests.

Patient consent Not required.

Provenance and peer review Not commissioned; externally peer reviewed. Data sharing statement № additional data are available.

Open access This is an open access article distributed in accordance with the Creative Commons Attribution Non Commercial (CC BY-NC 4.0) license, which permits others to distribute, remix, adapt, build upon this work non-commercially, and license their derivative works on different terms, provided the original work is properly cited, appropriate credit is given, any changes made indicated, and the use is non-commercial. See: http://creativecommons.org/licenses/by-nc/4.0/. 


\section{REFERENCES}

1. Tanios MA, Nevins ML, Hendra KP, et al. A randomized, controlled trial of the role of weaning predictors in clinical decision making. Crit Care Med 2006;34:2530-5.

2. Esteban A, Anzueto A, Frutos F, et al. Characteristics and outcomes in adult patients receiving mechanical ventilation: a 28-day international study. JAMA 2002;287:345-55.

3. Torres A, Gatell JM, Aznar E, et al. Re-intubation increases the risk of nosocomial pneumonia in patients needing mechanical ventilation. Am J Respir Crit Care Med 1995;152:137-41.

4. Thille AW, Richard JC, Brochard L. The decision to extubate in the intensive care unit. Am J Respir Crit Care Med 2013;187:1294-302.

5. Heunks LM, van der Hoeven JG. Clinical review: the ABC of weaning failure--a structured approach. Crit Care 2010;14:245.

6. Zapata L, Vera P, Roglan A, et al. B-type natriuretic peptides for prediction and diagnosis of weaning failure from cardiac origin. Intensive Care Med 2011;37:477-85.

7. Papanikolaou J, Makris D, Saranteas T, et al. New insights into weaning from mechanical ventilation: left ventricular diastolic dysfunction is a key player. Intensive Care Med 2011;37:1976-85.

8. Demoule A, Jung $B$, Prodanovic $\mathrm{H}$, et al. Diaphragm dysfunction on admission to the intensive care unit. Prevalence, risk factors, and prognostic impact-a prospective study. Am J Respir Crit Care Med 2013;188:213-9.

9. Levine S, Nguyen T, Taylor N, et al. Rapid disuse atrophy of diaphragm fibers in mechanically ventilated humans. $N$ Engl J Med 2008;358:1327-35

10. Vassilakopoulos T, Petrof BJ. Ventilator-induced diaphragmatic dysfunction. Am J Respir Crit Care Med 2004;169:336-41.

11. Kim WY, Suh HJ, Hong SB, et al. Diaphragm dysfunction assessed by ultrasonography: influence on weaning from mechanical ventilation. Crit Care Med 2011;39:2627-30.

12. Ali ER, Mohamad AM. Diaphragm ultrasound as a new functional and morphological index of outcome, prognosis and discontinuation from mechanical ventilation in critically ill patients and evaluating the possible protective indices against VIDD. Egypt $J$ Chest Dis Tuberc 2017;66:339-51.

13. Dres M, Dubé BP, Mayaux J, et al. Coexistence and Impact of Limb Muscle and Diaphragm Weakness at Time of Liberation from Mechanical Ventilation in Medical Intensive Care Unit Patients. Am J Respir Crit Care Med 2017;195:57-66.

14. Whiting PF, Rutjes AW, Westwood ME, et al. QUADAS-2: a revised tool for the quality assessment of diagnostic accuracy studies. Ann Intern Med 2011;155:529-36.

15. Higgins JP, Thompson SG. Quantifying heterogeneity in a metaanalysis. Stat Med 2002;21:1539-58.

16. Doorduin J, van Hees HW, van der Hoeven JG, et al. Monitoring of the respiratory muscles in the critically ill. Am J Respir Crit Care Med 2013;187:20-7

17. Llamas-Álvarez AM, Tenza-Lozano EM, Latour-Pérez J. Diaphragm and lung ultrasound to predict weaning outcome: systematic review and meta-analysis. Chest 2017;152:1140-50.

18. Zambon M, Greco M, Bocchino S, et al. Assessment of diaphragmatic dysfunction in the critically ill patient with ultrasound: a systematic review. Intensive Care Med 2017;43:29-38.
19. Jiang JR, Tsai TH, Jerng JS, et al. Ultrasonographic evaluation of liver/spleen movements and extubation outcome. Chest 2004;126:179-85.

20. Hu S, Zhou S, Wu D, et al. The predicting value of diaphragm ultrasound for weaning. Acta Universitatis Medicinalis Anhui 2016;51:673-7.

21. Spadaro S, Grasso S, Mauri T, et al. Can diaphragmatic ultrasonography performed during the T-tube trial predict weaning failure? The role of diaphragmatic rapid shallow breathing index. Crit Care 2016;20:305-16.

22. Farghaly S, Hasan AA. Diaphragm ultrasound as a new method to predict extubation outcome in mechanically ventilated patients. Aust Crit Care 2017;30:37-43.

23. $\mathrm{Xu} \mathrm{S}, \mathrm{Bu} \mathrm{Z}$, Pan C, et al. Diaphragm excursion as a predictor of difficult weaning from mechanical ventilation in patients with severe chronic obstructive pulmonary disease. Chinese Journal of Critical Care Medicine 2017;37:49-53.

24. Vassilakopoulos T, Zakynthinos S, Roussos C. The tensiontime index and the frequency/tidal volume ratio are the major pathophysiologic determinants of weaning failure and success. Am J Respir Crit Care Med 1998;158:378-85.

25. Capdevila X, Perrigault PF, Ramonatxo $M$, et al. Changes in breathing pattern and respiratory muscle performance parameters during difficult weaning. Crit Care Med 1998;26:79-87.

26. Mead J. Functional significance of the area of apposition of diaphragm to rib cage [proceedings]. Am Rev Respir Dis 1979;119:31-2.

27. Gong J, Zhang B. A comparative study on predicting outcome of ventilator weaning by diaphragmatic excursion,spontaneous breathing trial and rapid shallow breathing index. Trauma \& Critical Care Medicine 2016;4:133-7.

28. Carrie C, Gisbert-Mora C, Bonnardel E, et al. Ultrasonographic diaphragmatic excursion is inaccurate and not better than the MRC score for predicting weaning-failure in mechanically ventilated patients. Anaesth Crit Care Pain Med 2017;36:9-14.

29. Vivier E, Mekontso Dessap A, Dimassi S, et al. Diaphragm ultrasonography to estimate the work of breathing during noninvasive ventilation. Intensive Care Med 2012;38:796-803.

30. Ferrari G, De Filippi G, Elia F, et al. Diaphragm ultrasound as a new index of discontinuation from mechanical ventilation. Crit Ultrasound J 2014;6:8-14

31. De Jonghe B, Bastuji-Garin S, Durand MC, et al. Respiratory weakness is associated with limb weakness and delayed weaning in critical illness. Crit Care Med 2007;35:2007-15.

32. Supinski GS, Callahan LA. Diaphragm weakness in mechanically ventilated critically ill patients. Crit Care 2013;17:R120-37.

33. Dubé BP, Dres M, Mayaux J, et al. Ultrasound evaluation of diaphragm function in mechanically ventilated patients: comparison to phrenic stimulation and prognostic implications. Thorax 2017;72:811-8

34. Zhang Z, Xu X, Ni H. Small studies may overestimate the effect sizes in critical care meta-analyses: a meta-epidemiological study. Crit Care 2013;17:R2-11.

35. DiNino E, Gartman EJ, Sethi JM, et al. Diaphragm ultrasound as a predictor of successful extubation from mechanical ventilation. Thorax 2014;69:431-5. 